amentia; that in 16 out of the 94 cases of idiopathic epilepsy the patients were imbeciles, while 16 out of the 35 cases of organic lesion of the brain or primary amentia were imbecile. When a child has neither gross organic abnormality nor primary amentia but takes fits, these may have very different etiology and prognosis, and call for very different treatment. Epileptiform attacks forming part of the picture of spasmophilia-Group 3are associated with rickets; they are intimately connected with the metabolism of calcium and phosphorus, are controlled by calcium injection, and can be prevented and cured by means of vitamin D. The ultimate prognosis here, for the mental condition also, is good.

Fits that may be classified under Group 4 are of certain types-for example, (a) infantile eclampsia, (b) an occasional fit or fits, (c) pyknolepsy, and $(d)$ characteristic idiopathic epilepsy. In all, the presence or absence of an essential or idiopathic epilepsy may be conjectural for a time, but in none may the matter be lightly regarded. Although we have definite signs in spasmophilia for an independent diagnosis, and a definite exciting cause, the importance of a possible underlying diathetic trend towards convulsion is even here hard to evaluate.

When dealing with cases of infantile eclampsia we are on even less sure ground. Under this term I include the type of convulsions occurring during the first few weeks of life, usually in boys, where there is no apparent cause and often no digestive disorder, where the fits are of the nature of short general convulsions repeated at brief intervals, where other treatment is unavailing, but where temporary chloralization produces a permanent cure. Whether or not we have here to deal with an anaphylactic phenomenon is not known.

With a child who has an occasional fit we cannot altogether dismiss the possibility of an underlying vice of nature, provided there is no obvious cause, such, for example, as alkalosis. There is no certain test. But family and personal history, precedent or accompanying symptoms, the general physical and mental condition, may all go far to confirm a diagnosis in one or other direction.

In the rare type of case described as pyknolepsy we find that a previously healthy child of about 4 or 5 years of age suddenly begins to have fits of the nature of mild petit mal, which occur very regularly and usually very frequently from the onset. There may be as many as a hundred daily. As they commence, so they continue. No treatment has any beneficial effect; clinically striking is the fact that in spite of long-continued frequent attacks there is no mental impairment and no physical ill-health. After a variable number of months or years the attacks cease as suddenly as they began.

In striking contrast to such a course are most cases of ordinary idiopathic epilepsy; here the treatment consists essentially in attempts to restore nervous tone, and this demands attention to all bodily functions, particularly digestion and excretion. Chloralization is not successful, but the regular and long-continued administration of bromide and probably luminal is of the utmost importance. It is also necessary to establish intelligent control of instinct and emotions, to develop good habits, and to inculcate obedience, reticence, and modesty. Careful guidance of interest, occupation, and scholastic instruction is helpful. In some cases many of the peculiarities of the epileptic are due rather to his training, or rather spoiling, than to his weakness itself. The child is commonly not only a victim of a neuropathic heredity, but suffers also from a psychopathic home environment. Loose control is highly deleterious: the more averagenormal his training the better. The satisfactory care of chronic nervous or spasmodic conditions, especially of chorea, asthma, and epilepsy, demands the co-operation of parents, nurses, teachers, and doctors, and continuity of treatment for a long time.

1 Hall, Stanley: Youth, 1907.

RFFERENCES

Eckert: Zeit. $f$ Kinderleilk., 1924, xxxvii, 245

3 Graham and Anderson: Lancet, 1924, i, 1307.

4 McNeil: Edinburgh Med. Journ. 1924, 1307.

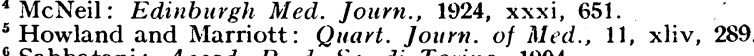

Sabbatani: Accad. R. d. Sc. di Torino, 1904.

7 Percival: British Medical Journal, 1927, ii, 783.

${ }^{8}$ Eppinger and Hess: Journ. of Nerv. and Ment. Dis., 1914, xli, and 1915, xlii.

9 McDougall, William: An Outline of Psychology. Methuen, 1926.

10 Meyer:Zeit. $f$ Kinderleill 1929 , xlvii, 560.

11 Mackenzie, Ivy: Glasgow Med. Journ., 1915, 1xxxiii, Nos. 4, 5, and 6 .

\section{SUBPHRENIC ABSCESS}

BY

W. D. DOHERTY, M.CH., F.R.C.S.

SENIOR SURGICAI, REGISTR.IR AND TUTOR, GUY'S HOSPITAL, AND SURGEON, HAMPSTEAD HOSPITAL FOR CHILDREN AND

R. P. ROWLANDS, M.S., F.R.C.S. SLRGEON, GUY'S HOSPITAL

A subphrenic, or subdiaphragmatic, abscess is a limited collection of pus just below the diaphragm. Frequently gas, either from a perforated viscus or as a product of gas-forming bacteria, is also present in the abscess cavity.

H. L. Barnard, ${ }^{1}$ in his excellent paper upon this subject, analysed seventy-six consecutive cases, and classified subphrenic abscesses as follows:-

Intraperitoneal : right anterior, right posterior, left anterior, left posterior; of these, anterior abscesses are more common than posterior, especially on the left side.

Extraperitoneal: right, left; of these, the right-sided is mi.sh more frequent.

The falciform ligament is the dividing line between the right and left intraperitoneal abscesses, and the lateral ligaments of the liver separate the anterior from the posterior varieties. The right extraperitoneal space under the diaphragm is small, and is limited by the coronary ligament of the liver; the left extraperitoneal space is near the upper pole of the kidney. Extraperitoneal abscesses, however, may travel upwards behind the peritoneum from the colon, appendix, or kidney, pushing the peritoneum forward and eventually occupying the large space under the diaphragm. Sometimes a subphrenic abscess extends downwards into the subhepatic space on either side of the middle line. In the present series of 49 cases, 36 occurred on the right side, and 13 on the left.

CAUSES
Micro-organisms may travel by the blood stream from any part of the body and cause a subphrenic abscess, but the abdominal viscera are naturally the sources of most abscesses in this position, and of these the stomach and the appendix originate the large majority. The liver, gall-bladder, and bile ducts together contribute the next largest group of cases. Here the infection is usually spread direct from a perforated viscus, but it may be carried by the portal veins or by the lymphatics. Diseases of the chest, especially of the lungs, cause a few subphrenic abscesses; diseases and injuries of the chest wall, particularly of the ribs, are also etiological factors in a few cases. Either systemic or portal pyaemia may give rise to a subphrenic abscess. Many different organisms have been found in these abscesses, notably the Staphylococcus aureus, streptococcus, pneumococcus, $B$. coli communis, $B$. pyocyaneus, and $B$. typhosus. Subphrenic abscesses are most frequent between the ages of 20 and 50, and they are distinctly commoner in men than in women. 
An analysis of 49 consecutive cases at Guy's Hospital shows the following causes:

Perforated gastric and duodenal ulcers* Acute appendicitis

Ilood-borne

Blood-borne $\ldots$...

Acute cholecystitis

Carcinoma of colon...

Carcinoma of stomach

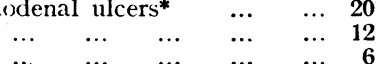

$\cdots+\cdots$

$\cdots+\cdots$, phrenic abscesses found post mortem as part of a general peritonitis.

\section{Symptoms}

Fever, remittent or intermittent, and with or without rigors and associated abnormal physical signs at the base of the chest, suggest a subdiaphragmatic abscess, especially if there is a known source of infection such as a recent appendicitis or perforation of a gastric or duodenal ulcer. Dullness over the lower part of the chest is the most constant local sign, and the upper limit of this is often dome-shaped. This dullness is continuous with that of the liver or spleen. Sometimes there may be three or four zones of varying resonance at the base of the chest, the liver or splenic dullness being continued upwards to that over the collection of pus under the diaphragm. Just above this there may be a tympanitic note over a collection of gas above the pus, higher up a dull area indicating pleural effusion, and still higher the normal resonance of the lung. Although there may be no pulmonary signs at first, these develop later, due to the spread of congestion and inflammation to the pleura and lung. Sooner or later there may be pleurisy with a "rub," followed by effusion; there may be signs also of inflammation and perhaps some collapse at the base of the lung with rales and rhonchi, and particularly deficient entry of air and tubular breathing. Lower down, over a collection of gas below the diaphragm, there may be the coin sound or bruit d'airain. Leucocytosis is an important and almost constant sign. In late cases there may be bulging of the corresponding side of the chest, and later still oedema and even pointing of the abscess generally below the costal margin, either in front or behind.

\section{Diagnosis}

The diagnosis of this condition is unfortunately difficult, and is too often seriously delayed. A good radiographic examination is invaluable; often there is shown a characteristic local elevation with fixation of the dome of the diaphragm. In some cases a collection of gas above the pus is also shown upon the screen. It must not be forgotten, however, that the retraction of a fibrosing lung may pull the diaphragm up so much and in such a way as closely to simulate the shadow of a subphrenic abscess. Similarly the stomach has been mistaken for a subphrenic abscess on the left side. In the hope of settling the diagnosis a large and long exploratory needle is generally relied upon, but unfortunately the needle often fails us, either getting blocked by thick lymph or missing the abscess. It failed in eleven out of eighteen cases so tried by Barnard. There is a far more reliable method of proving the presence or absence of a subdiaphragmatic abscess, and when in doubt in these grave cases, where a prompt decision is absolutely necessary to save life, this method should always be used. Under general or local anaesthesia an incision just large enough to admit the hand is made in the epigastrium, and the hand is passed in all directions above the liver. Within a few minutes the diagnosis is settled, and an abscess, either under the diaphragm or in the liver, is not only found but accurately located; it can then be safely and quickly drained below the pleural reflection and extraperitoneally, thus avoiding the grave risks of pneumothorax, empyema, and peritonitis.
Upon three occasions, after being asked to drain a subphrenic abscess, one of us used the epigastric incision, and proved that no abscess existed, although the radiograms had shown shadows which were thought to be typical. It is often almost impossible to distinguish empyema from a subdiaphragmatic abscess. In both, the cupola of the diaphragm is fixed, but in the former it is depressed, and in the latter elevated in a characteristic way, leaving the costophrenic angle transparent (Fig. 1).

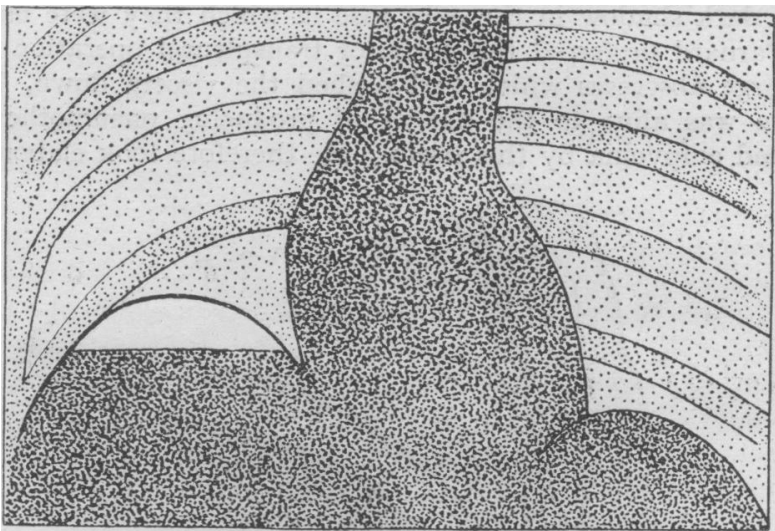

FIG. 1.-Diagram showing the radiographic appearances of a gas-containing subphrenic abscess on the right side. The right cupola of the pushed up and

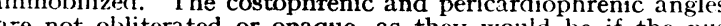
had been above the diaphragm.

The exploring needle does not help in the distinction. Not uncommonly, empyema complicates a subphrenic abscess.

\section{Prognosis}

In our experience, subphrenic abscess is much rarer than it used to be. Over a period of ten years since the war, the Guy's Hospital surgical reports record forty-nine cases, an average of under five cases a year. This is almost certainly due to earlier diagnosis and operation for perforated peptic ulcer and acute appendicitis, and to the better packing off of the subphrenic space and the avoidance of irrigation during these operations. The frequent adoption of the Fowler position has also helped.

\section{TREATMENT}

Without operation there is very little hope of recovery, although the abscess may drain imperfectly through the lung into one of the hollow abdominal organs or through the skin. The few patients who survive this tedious process are apt to become chronic invalids. The main ideal of successful operation is efficient drainage without risks either of pulmonary complications or of peritonitis. To attain this ideal every effort should be made to drain the abscess at its lower part, below the pleural, reflection, and extraperitoneally.

The anterior abscesses, which are fortunately most common, often descend into the epigastrium or hypochondrium. They can be drained through an incision below the costal margin, but a counter-incision in the loin is sometimes also necessary. Posterior abscesses may be drained through the thorax, either by a subpleural or transpleural route. The cases cited below illustrate the frequency with which pulmonary complications follow the transpleural route, yet this still appears to be the common method of approach. It is interesting in this connexion to review Barnard's lines of treatment.

Barnard did not advocate early operation upon these abscesses, but preferred in most cases to delay intervention until the abscess had enlarged considerably and was well shut off. His reasons for doing so were that as the abscess enlarged the diaphragm was driven up, and the 
lower part of the pleural cavity was obliterated or closed by adhesions. The abdominal viscera, on the other hand, were pushed down, so that when the transpleural operation was chosen the abscess could be opened through a lower spot in the thoracic wall. It is apparent from Barnard's case reports that the diagnosis of subphrenic abscess was not made until a late stage of the condition. He says that in fifty out of seventy-six consecutive cases an abdominal swelling was noticed as one of the signs of the abscess. Again, $x$ rays demonstrated the abscess as a dark shadow in the only case in which they were used. Barnard preferred the posterior transpleural operation for large abscesses which had pushed the viscera down, but he was alive to the danger of producing pneumothorax and empyema by this route. He therefore took elaborate

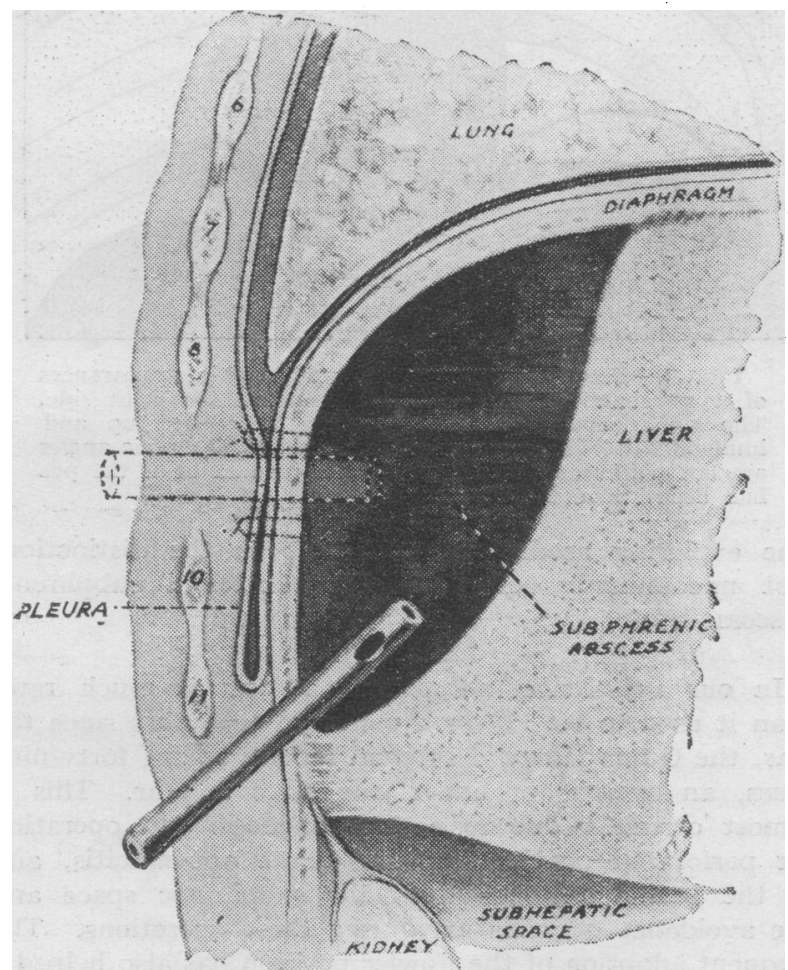

FIG. 2.-Subpleural and retroperitoneal drainage of a subphrenic abscess; a part of the twelfth rib has been removed, and the lower tube has been inserted through a horizontal incision in the diaphragm below the pleura. The upper shadowed tube passes through the pleura. This method is to be avoided, because pneumothorax and infection of the pleura may result even after all precautions have been taken to avoid them, and even when the pleura is adherent at the time of the operation.

precautions to prevent these complications. He got an assistant to push up the liver whilst he was incising the pleura. Then he sutured the pleura on the surface of the diaphragm to the parietal pleura, even although adhesions were present. Lastly, having incised the diaphragm, he sutured the latter to the intercostal muscles.

At the present time subphrenic abscesses are recognized at a comparatively early stage by improved methods of radiography, etc., and they call for treatment before the pleural cavity has become obliterated and the viscera pushed down to any great extent. It is particularly important to avoid the transpleural operation in these early cases, but even in late cases it is not so safe as the subpleural operation, pneumothorax with pleural infection having often followed, even when the lower part of the pleura has appeared to be safely obliterated either by nature or by art. Therefore the exploring needle should be inserted below the pleural reflection, which runs obliquely about an inch above the lower costal margin. The needle should be passed obliquely upwards and in- wards. If pus be found the incision should follow the needle, the diaphragm being exposed and a transverse incision made through it below the pleura. Generally it is necessary to remove a portion of one or more ribs below the pleura in order to get adequate room (Fig. 2). Nather and Ochsner remove the whole of the last rib, but it is enough, and safer, to excise only the distal half, because the pleura often extends below the neck of the last rib, where it is liable to be wounded. The transverse incision through the diaphragm is made at the level of the first lumbar spine, and therefore nearly always well below the pleura. The peritoneum lining the diaphragm is carefully separated and pushed forward, and the closed points of long dressing forceps are passed just below and along the needle into the abscess. The forceps are opened gently to enlarge the opening, and a large rubber tube is inserted and stitched to the skin.

\section{MORTALITY}

Without operation the death rate of subphrenic abscess is over 85 per cent. With operation it used to be over 50 per cent.; in recent years, however, it has been reduced to about 25 per cent. Before long it should be further reduced by earlier diagnosis and better operations, especially with the avoidance, whenever possible, of pulmonary complications.

\section{CASE I}

R. B., aged 20, was admitted to Guy's Hospital on March $30 \mathrm{th}, 1930$. On the 24 th he had had pain in the region of the umbilicus, associated with nausea and vomiting; on the 25th the pain had travelled to the right iliac fossa and had got steadily worse; on the 30th the pain was excessive; the bowels had not opened for five days: On examination the abdomen was rigid all over, and particularly on the right side; there was no special tenderness in the right iliac fosst. Rectal examination showed tenderness in all directions. His temperature was $103^{\circ} \mathrm{F}$., and pulse 120 ; the tongue was dry and brown. The abdomen was opened the same day through a lower right paramedian incision, and a gangrenous appendix was removed. The peritoneum was full of pus. The pelvis and the right iliac regions were drained through stab wounds. He did fairly well until April 12th, when his temperature rose to $102^{\circ}$. The right chest was rigid, and there was dullness over the lower part of it. On the 13th, the eighth space, on the right side beneath the axillary line, was aspirated and three-quarters of a pint of foul pus, containing Bacillus coli and streptococci, was removed. The following day another pint of similar pus was removed, and as there still appeared to be more, further operation was undertaken, a piece of the eighth rib removed, and a subphrenic abscess drained. The diaphragm was found to be firmly adherent to the parietal pleura. On April 19th he collapsed again, and was found to have a pneumothorax; air was sucking in and out with each breath, around the drainage. A larger drain was inserted and sealed to the thoracic wall with a rubber dam. Negative pressure was maintained by suction with Sprengel's pump, but he died the following day. At the subsequent examination the abscess cavity was found almost empty, and there was no empyema on either side.

\section{CASE II}

A man, aged 45, was admitted to Guy's Hospital on April 22nd, 1930, for a perforated duodenal ulcer with extravasation. The perforation was closed and the wound was drained, and everything went satisfactorily until about three weeks later, when the temperature went up and remained remittent, and about $101^{\circ} \mathrm{F}$. in the evening. The man made no complaint of pain in his back, but there was dullness at the right base. The radiograms did not reveal any bulging up of the diaphragm, but only fixation of the right cupola. The leucocyte count was 20,000. A physician saw him and was a little uncertain as to the exact cause of the sumptoms. A needle was inserted in the right pleura, without finding any pus; only a little clear fluid was withdrawn.

Under an anaesthetic on May 22nd, the patient lying on his left side, a large needle was passed obliquely upwards and forwards from behind and below the last rib. At the second 
venture offensive brown pus was found. Then, through an oblique incision, the terminal half of the last rib was removed and the diaphragm cut horizontally; it was thickened and inflamed. Forceps were passed upwards and forwards aleng the needle into the abscess cavity, which was the size of an orange, and lying chiefly behind the right lobe of the liver. It was drained through a large tube, and the patient made a good and rapid recovery.

\section{CASE III}

T. B., a male aged 59, had had indigestion and attacks of vomiting for years. On November 1st, 1922, he was seized with sudden severe abdominal pain, accompanied by vomiting, and was admitted to Guy's Hospital on November 4th. The right upper quadrant of the abdomen was rigid, and there were signs of compression of the base of the right lung. The $x$-ray report stated: "Right diaphragm is greatly elevated and completely immobile. Left diaphragm moves well. Gas bubble above right lobe of liver." This abscess was explored transpleurally by resecting the tenth rib in the mid-axillary line, four and a half pints of fluid being removed. The patient died on Becember 11 th, and at the post-mortem examination a large right empyema and collapsed lung were found. The subphrenic abscess was secondary to a perforated duodenal ulcer.

\section{CASE IV}

I. G., aged 33. A gastric ulcer was operated upon seven hours after perforation, suture of ulcer being done with suprapubic drainage. Eight days later pain was complained of below the left costal margin, and examination proved a "rub" at the left base. A straw-coloured fluid was aspirated. On the eleventh day a left subphrenic abscess was drained by incision below the last rib. On the fourteenth day there was rib resection for left-side empyema; the patient died on the twenty-eighth day.

\section{CASE V}

C. McC., aged 62 years, was admitted to Guy's Hospital on May 2nd, 1925, with a fifteen-hour history of acute pain in the upper abdomen. Laparotomy revealed a perforated duodenal ulcer. Suture with drainage of the peritoneum was performed. Three weeks later he had pyrexia with pain over the lower ribs on the right side. The white blood count was 20,000. $X$-ray report: "Right leaf of diaphragm is slightly elevated, movements greatly diminished. Slightly indefinite opacity at right base suggestive of pleural thickening, but no effusion seen. Left base and left diaphragm show no abnormality." Pus having been found by an exploring needle on June 11 th a portion of the eleventh rib was resecied on the right side; the lower margin of the pleura was found and sutured to the diaphragm. The diaphragm was then opened subpleurally and the abscess drained. He made a satisfactory recovery, and was discharged a fortnight later.

\section{CASE VI}

K. S., aged 38, was admitted to Guy's Hospital on September 12th, 1927, with fourteen days' history of pain in the left loin, with pyrexia. There was tenderness in the left loin and dullness at the left base of the lung, but $x$ rays showed no abnormality. An exploring needle inserted through the tenth space located pus apparently below the diaphragm. A portion of the eleventh rib was resected and the pleural cavity opened; no pus was present. The pleura was closed and the diaphragm incised and a subdiaphragmatic abscess drained. One month later the pyrexia was still present, and pus was found by an exploring needle in the left pleural cavity; this was drained through the original wound. After a prolonged convalescence the patient left the hospital with a discharging sinus.

\section{CONCLUSIONS}

A review of the causes, symptoms, and diagnosis of the subphrenic abscess is given.

The treatment is discussed, and the posterior subpleural method of approach is advocated in order to avoid pulmonary complications.

Cases are cited illustrating the complications following the transpleural route. Cases II and V show the advantages of the subpleural route.

${ }^{1}$ H. L. Barnard: Brilish Medical Journal, 1908, i, 371, 429

\section{GENERAL OBSERVATIONS ON SEA-SICKNESS AND THE LABYRINTHINE THEORY*}

\author{
BY
}

\section{T. GWYNNE MAITLAND, M.A., B.Sc., M.D., D.PhrL。} MEDICAL SUPERINTENDENT, CUNARD COMPANY

The British nation, eminently seafaring, is conspicuous for its neglect of the study of sea-sickness. In consequence of this ignorance there is as much diversity of opinion about cause as there is about treatment. The very prevalent view that sea-sickness is a matter of mere nerves arises from the observations that vomiting sometimes occurs before the passenger has embarked, and also that sea-sickness is apparently so often controlled by suggestion. There are other views equally superficial which overlook the primary cause because of some casual feature which is unduly prominent. So it is with treatment. The layman, in the person of the old traveller or the bedroom steward, is always prepared with an "infallible remedy." Even the medical man, at sea as on shore, may have partial success with a favourite drug, and, turning a blind eye to failures, may become stereotyped in his advice. The underlying factors are altogether too inconstant for certitude. Personal adaptability, the subsidence of winds and waves-any one or all may make a remedy appear effective. Thus it is advisable that the would-be voyager, when urged to try so many " personally recommended cures," should bear in mind the poverty that lies in such wealth.

\section{Conditions at Sea}

It is exceptional to have seas continuing rough for a trip of six days. It is not incommon to hear sailors speak of a wind as "blowing itself out"; rough days alternate with calm days; all the greater care is needed, therefore, in waxing enthusiastic over a specific remedy. The weather may have changed, the sea subsided, the first attack of vomiting have corrected the overtaxed digestion, or one's sea legs may have come to the rescue. For these reasons it is necessary to appraise all the accessory aids as nearly as possible in order to judge how far any particular remedy has contributed towards recovery. Care must also be exercised as to the denotation of sea-sickness in order to be quite sure that we are considering sea-sickness and not sickness at seacases of mere " nerves," those who are " sea-sick" when they step on the gangway or even (as in one case) when in sight of the sea, those who vomit merely in imitation or in consequence of the odour of vomiting, all in fact, belonging to the readily suggestible, but who do not properly come within the scope of the inquiry.

\section{The Ship's Movement}

Quite a large number of old travellers (ship captains among them) never acquire complete adaptability to ship movement. One ship surgeon of fourteen years' standing never püt out to sea without, for the first two days, headache, mental confusion, and nausea, which went on to vomiting if the sea became rough. Long familiarity with one type of ship, in susceptible persons, may give undue confidence in their capacity as good sailors, only to have this rudely shattered as in the case of so many " old salts" when transferred during the war from big battleships and big liners to destroyers and trawlers. One may venture to say that with the exception of certain rare and mostly pathological cases, all normally constituted people are susceptible to some definite type of movement. Nevertheless sea-sickness has not the terrors for passengers it had in old times. Our parents may have been hardier, but the descriptions of crossings

* Read in the Section of Medicine at the Anmual Meeting of the British Medical Association, Winnipeg, 1930. 